\title{
Study of high frequency MHD modes from ECE radiometer in Tore Supra
}

\author{
D. Elbeze ${ }^{1}$, Z.O. Guimarães-Filho ${ }^{2}$, D. Molina ${ }^{1}$, J.-L. Ségui ${ }^{1}$, S. Benkadda ${ }^{2}$, N. Dubuit $^{2}$, X. Garbet $^{1}$ \\ and R. Sabot ${ }^{1}$ \\ ${ }^{1}$ CEA, IRFM, F-13108 Saint Paul Les Durance, France. \\ ${ }^{2}$ Aix Marseille Univ., IIFS/PIIM, Marseille, France.
}

\begin{abstract}
Tore Supra ECE diagnostic has been recently upgraded to study MHD modes driven by energetic particles up to $400 \mathrm{kHz}$. To improve the measurement sensitivity, the ECE signals of the 32 channels radiometer were amplified just below the saturation limit and sources of noise were investigated in order to keep it as low as possible. With such an improvement, fast particle driven modes with frequencies up to $200 \mathrm{kHz}$ were detected. A 4-channel correlation ECE system using YIG filters with tuneable frequency was also installed. It allows fine radial scans of MHD modes and correlation length measurements. For the two kinds of YIG filter in use, the minimum frequency separation between two ECE channels that could be achieved was established measuring the correlation coefficient between the respective radiation noises. Finally, by modelling the ECE radiometer taking into account the antenna radiation pattern and the vertical position of the ECE beam relative to the plasma centre we improved the data analysis tools, thus giving a better determination of the phase radial structure of ECE oscillations. The poloidal structure of MHD modes can then be identified from ECE data and, for off axis ECE lines of sight, the direction of the plasma rotation can also be determined. This method allows identifying the occurrence of an inverse cascade of electron fishbone modes ranging from $m / n=4 / 4$ to $1 / 1$ ( $m$ and $n$ are the poloidal and toroidal mode numbers, respectively) which appears in lower hybrid current drive plasmas.
\end{abstract}

\section{ECE diagnostic improvements}

At Tore Supra, the radial profile of electron temperature $T e$ is routinely measured by an ECE radiometer with 32 equatorial line of sight channels and $1 \mathrm{GHz}$ sampling frequency. The spatial arrangement of these 32 channels allows for radial resolution of $2.5 \mathrm{~cm}$ and transverse resolution of $8 \mathrm{~cm}$. Depending on the magnetic field, more of half of the plasma radius is usually probed by the diagnostic (figure 1).

Three separate ECE systems give Te profiles and fluctuations, as detailed in the following:

1. A slow acquisition system, at $1 \mathrm{kHz}$, delivers Te profiles for all the discharge duration with absolute values of $T e$ thanks to the in situ calibration [1].

2. A fast acquisition system, at $1 \mathrm{MHz}$, is used to observe Te fluctuations in particular those induced by magnetohydrodynamic (MHD) modes up to $400 \mathrm{kHz}$. 
3. A four-channel system with adjustable frequencies provides $T e$ fluctuation measurement with improved $\mathrm{S} / \mathrm{N}$ ratio using correlation of two adjacent channels probing the same plasma region with two different frequencies [2].

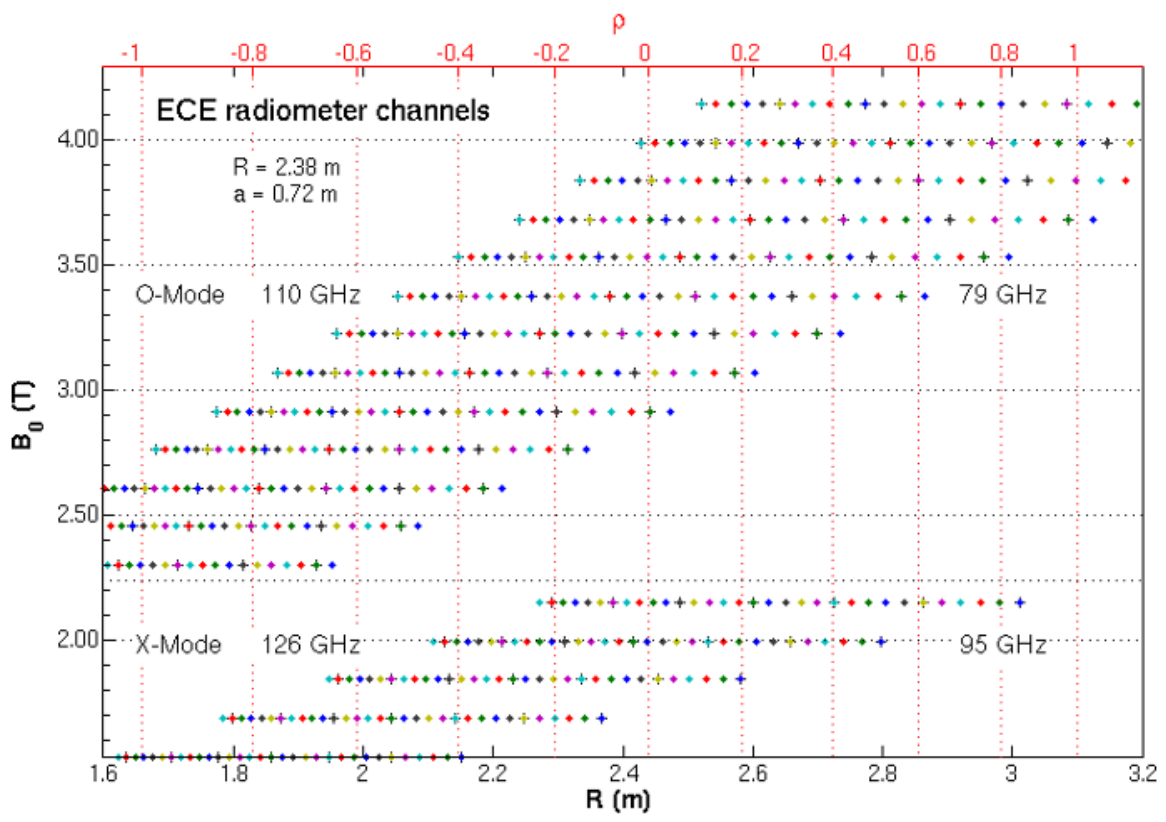

Fig. 1. Radial positions of the 32 ECE channels of the Tore Supra radiometer according to the toroidal magnetic field. As an indication, the top axis gives the normalized radius.

Fast ECE- In 2009, the fast acquisition system of the ECE radiometer was improved to detect high frequency MHD modes up to $400 \mathrm{kHz}$ during up to 7 seconds in all discharges. To detect small temperature perturbations $(\Delta T / T \sim 0.2 \%)$, the ECE signals were amplified just below the saturation limit for each channel and investigation of the sources of noise was performed to improve the $\mathrm{S} / \mathrm{N}$ ratio.

Correlation ECE- In 2010, two correlation ECE channels were added to the two existing ones [3]. The new YIG filters installed do not have the same frequency bandwidth, which is now $100-$ $200 \mathrm{MHz}$ instead of $40-80 \mathrm{MHz}$. A larger bandwidth improves the $\mathrm{S} / \mathrm{N}$ ratio [2] but the frequency range of the new kind of YIG filter is reduced to $4-18 \mathrm{GHz}$ instead of $2-18$. Therefore, a part of the probed region corresponding to $2-4 \mathrm{GHz}$ is now inaccessible. During the last experimental campaign, 2 kinds of YIG filter were tested to determine the minimum frequency shift between 2 adjacent channels ensuring uncorrelated ECE noise (Figures $2 \& 3$ ).

Analysis tools- Spectral analysis of ECE signals is adapted to the study of electron temperature ( $T e$ ) oscillations induced by MHD modes. The observation time is long (up to 7 seconds), which is suitable to track physical phenomena from signal spectrograms. Over such a long time: plasma equilibrium usually evolves, some perturbations of the additional heating can occur or periodic relaxation phenomena, such as sawtooth or giant oscillations, can lead to modifications of the mode frequencies. When $T e$ fluctuations are quite large, cross-correlations between two successive channels are used to improve the $\mathrm{S} / \mathrm{N}$ ratio. In addition, the $32 \mathrm{ECE}$ channels are useful to locate MHD-induced oscillations in the radial direction and determine their radial structure by measuring the relative phase between channels. 


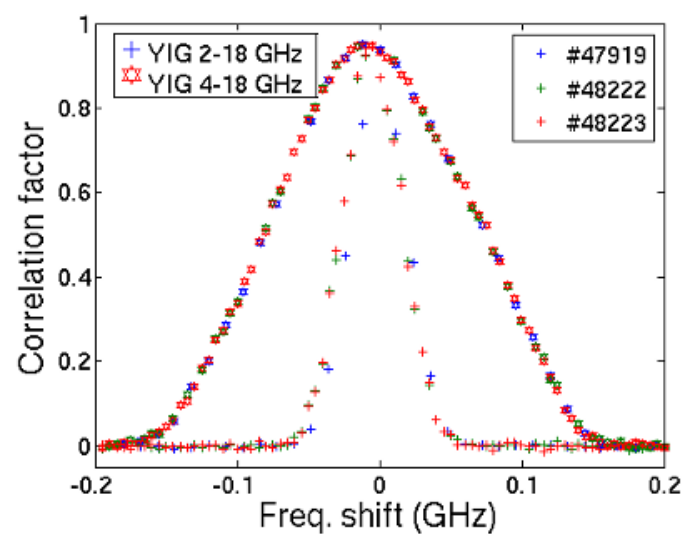

Fig. 2. Correlation coefficient as a function of the frequency shift between 2 channels for 2 kinds of YIG filters. One of the correlated channel frequencies is constant to $11.0 \mathrm{GHz}$ and the other one is randomly shifted from -0.2 to $0.2 \mathrm{GHz}$. These measurements were repeated during 3 Tore Supra discharges.

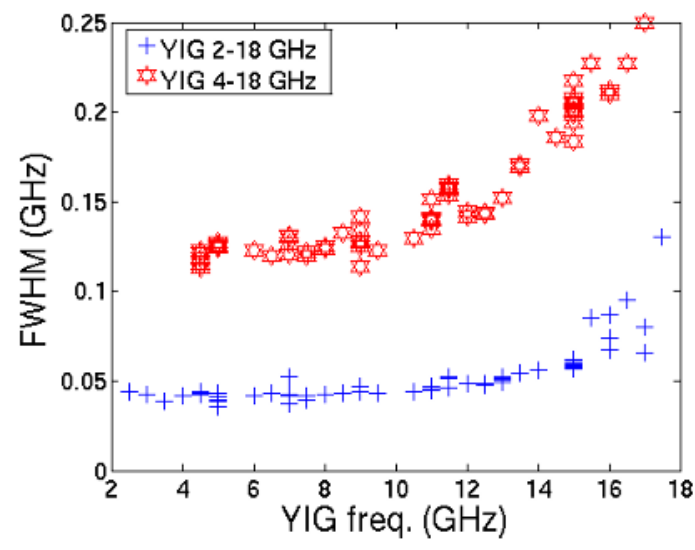

Fig. 3. Full width at half medium (FWHM) of the correlation coefficient profile as a function of the central frequency for 2 kinds of YIG filter. To ensure uncorrelated ECE noise $(-30 \mathrm{~dB}$ on the correlation coefficient), the frequency shift between the 2 adjacent channels should exceed twice the FWHM.

\section{Effect of the finite ECE beam size}

The radial profile of some core MHD modes measured with ECE can be quite complex, as presented in the left panel of Figure 4. In order to understand the origin of these complex structures we modeled the ECE diagnostic by considering the spatial resolutions in the radial and transversal directions. The central panel of Figure 4 presents the modeled $T e$ perturbations induced by modes with poloidal mode numbers, $\mathrm{m}=1,2$ and 3. The simulated MHD-induced Te perturbations are obtained by a simple model [4]:

$$
\tilde{T} e(r, \theta, t)=-\xi(r) \cdot \nabla T_{e} \cdot \cos (m \cdot \theta-\omega \cdot t)
$$

where $\omega=2 . \pi f$ is the angular frequency of the mode in the laboratory frame, $\xi(r)$ is the MHD displacement (which is a top flat function for a kink mode with $\mathrm{m}=1$ ) and $\nabla T_{e}$ is the equilibrium temperature gradient in the radial direction. The yellow rectangles in Fig. 4 illustrate the measured $T e$ oscillations of channels close to the magnetic axis, showing that the transversal resolution is much lower than the radial one.

The consequence in case of modes with $\mathrm{m}=2$, is that the average $T e$ oscillations in the central channel are in opposition of phase with respect to the phase shift peaks. Therefore, the radial profile of the measured $T e$ oscillations displays an inverted region close to the axis, as it can be seen in both the simulated profile (right panel) and the experimental one (left panel, Fig 4(c)). In case of $\mathrm{m}=3$ modes, two regions with the oscillations in opposition of phase with respect to the closest peak are observed (see Fig 4(b)) but these inversions does not occur in $\mathrm{m}=1$ modes (Fig 4(d)). Therefore, from the radial profile of the Te oscillations measured by ECE it is possible to know the poloidal mode number of core MHD modes [4]. In some Tore Supra shots with LHCD, modes identified as electron fishbones are sometimes observed [5-6]. Moreover, frequency jumps between modes with different mode-numbers are often observed in such discharges. By analyzing the radial profiles of the ECE measurements, one found that the sequence in mode numbers transition corresponds to: $\mathrm{m} / \mathrm{n}=4 / 4 \rightarrow 3 / 3 \rightarrow 2 / 2 \rightarrow 1 / 1[4]$. 
By using this model for the ECE measurements, it is also possible to observe that when the ECE line of sight is vertically shifted the direction of the poloidal projection of mode rotation can be determined by the phase shift of the Te oscillations [7]. In the case of Tore Supra, these methods based on ECE data analyses are particularly useful since the active cooling of the first wall components prevents the use of the magnetic coils to detect modes with frequencies above a few $\mathrm{kHz}$.
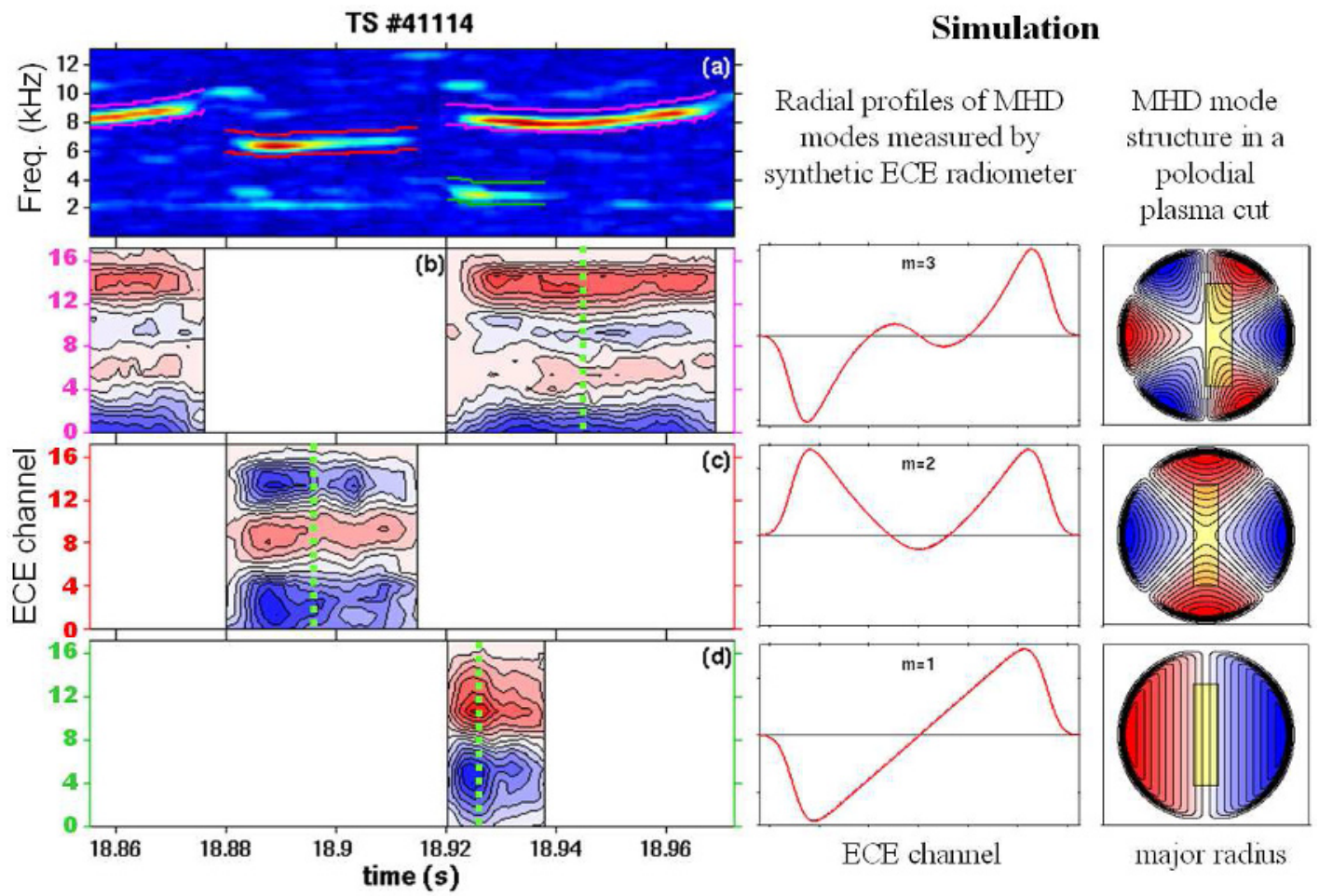

Fig. 4. Determination of the poloidal mode number from ECE measurements. The left panel presents the spectrogram of the Te oscillations (a); and the radial profiles of the $9 \mathrm{kHz} \mathrm{m} / \mathrm{n}=3 / 3$ (b), the $6 \mathrm{kHz}$ $\mathrm{m} / \mathrm{n}=2 / 2$ (c) and the $3 \mathrm{kHz} \mathrm{m} / \mathrm{n}=1 / 1$ (d) electron-fishbone modes obtained in a Tore Supra shot with LHCD . The right panel presents the simulated MHD induced Te oscillations for modes with poloidal mode numbers $m=1-3$ over the region probed by central ECE channels, which is imposed by the difference between the radial and the transversal resolutions. The central panel shows the correspondent radial profiles simulated by using the model proposed in references $[4,7]$.

\section{MHD mode observations}

In order to validate MHD codes and their predictions for ITER and future fusion reactor plasmas, simulations have to be compared to experiments by means of measurements that become more and more accurate and efficient. During the 2 last experimental campaigns at Tore Supra, several interesting MHD mode behaviours were observed and need to be confronted with physics interpretations.

For shot \#47313, several MHD modes with different frequencies coexist in the plasma core (figure 5). Key parameters of the discharge are:

- Additional heating injected power: 3.0 MW of ICRH and 5.0 MW of LHCD,

- Electron density: $410^{19} \mathrm{~m}^{-3}$,

- Total plasma current: 0.7 MA,

- $\quad$ Toroidal magnetic field: $3.8 \mathrm{~T}$. 
(a)

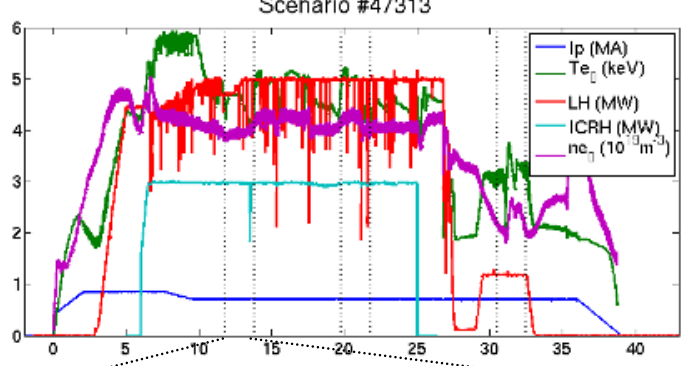

(b)
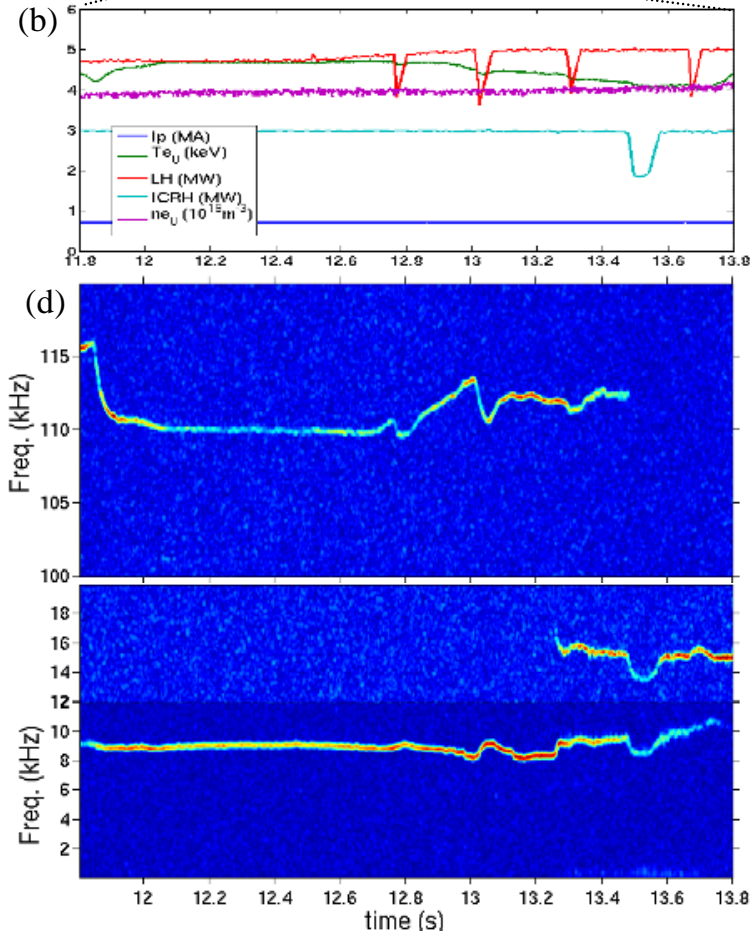

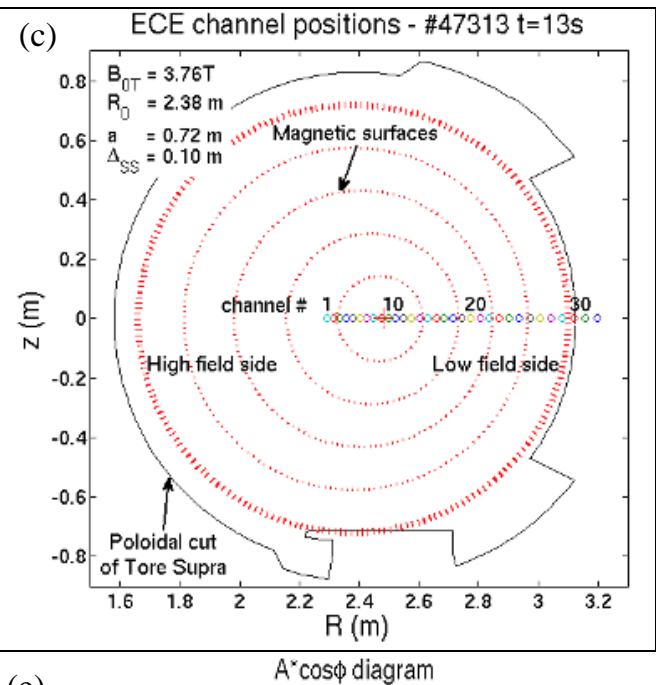

$(\mathrm{e})_{20}$
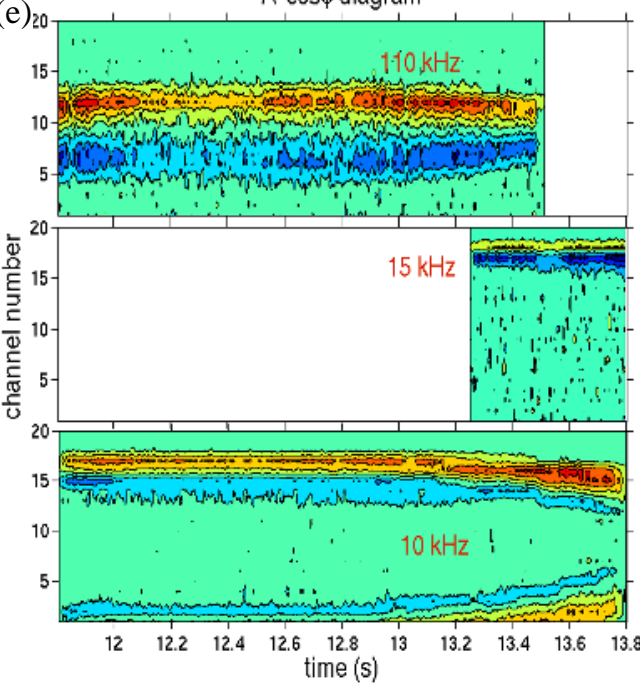

Fig. 5. (a) Plasma scenario of a high power discharge on Tore Supra with $8 \mathrm{MW}$ of added heating power and for which 3 fast ECE acquisition windows of 2 seconds were set-up. (b) Studied modes are on the $1^{\text {st }}$ ECE window. (c) Positions of the 32 ECE channels with respect to the plasma equilibrium for this discharge. (d) Cross-spectrograms of ECE channels showing the time-frequency evolution of 3 MHD modes. (e) Phase diagram $(A * \cos \phi)$ showing the phase shift between the various ECE channels. As the $y$-axis (channel numbers) represents the radial position, the time evolution of the poloidal structure can be studied for the 3 observed modes.

As shown on Figure 5 the 2 MHD modes with low frequencies $(10 \mathrm{kHz}$ and $15 \mathrm{kHz})$ observed are probably magnetic islands while the high frequency mode at $110 \mathrm{kHz}$ is an Alfvén Eigenmode. To improve the image contrast $(\mathrm{S} / \mathrm{N})$, cross-spectrograms are performed between two adjacent ECE channels [8]. The more intense channels for each frequency were used to produce figure 5-d. When the central temperature is stable $(12<\mathrm{t}<12.7 \mathrm{~s})$, frequencies are constant but after the slow decrease of the temperature, we can observe some frequency variations, which are not synchronous for the different modes. The brief diminution of ICRH power at $\mathrm{t}=13.5 \mathrm{~s}$ leads to a frequency drop and an intensity diminution of the island MHD modes, then confirming that ICRH directly affects the magnetic islands. 
The phase $(A * \cos \phi)$ diagram (figure 5-e) allows for the localisation of the mode with some information on its poloidal structure. For the 10 and $15 \mathrm{kHz}$ modes, the phase alternation is characteristic of a magnetic island. The poloidal mode number of the $10 \mathrm{kHz}$ mode is even as the Te oscillations in the low field side are in phase with the high field side ones. No information on the mode number can be obtained for the $15 \mathrm{kHz}$ mode because at $B_{T}=3.8 \mathrm{~T}$ the mode is too external to be probed at the high field side by the ECE radiometer. At Tore Supra, the observation of the plasma core by the ECE radiometer is better for a toroidal magnetic field $B_{T}$ of $3.5 \mathrm{~T}$ (Figure 1). The poloidal mode number of the high frequency mode is clearly odd and equal to 1 as discussed in section 2.

The position of the MHD modes gives interesting information. During the acquisition window, the $10 \mathrm{kHz}$ mode borders the internal high frequency mode. At $\mathrm{t}=13.25 \mathrm{~s}$, a $15 \mathrm{kHz}$ mode appears localised outside the $10 \mathrm{kHz}$ mode. In the meantime, the $10 \mathrm{kHz}$ mode is slowly drift inward, reflecting the modification of the q-profile (the position of the q surfaces) which started around $\mathrm{t}=12.9 \mathrm{~s}$. Inside the plasma core, the high frequency mode is also drifting towards the centre before fading at $\mathrm{t}=13.5 \mathrm{~s}$.

Understanding such phenomena could certainly help validate the physics models used to describe the interaction of MHD modes and energetic particles in the core region, which are necessary to make predictions to ITER.

\section{Acknowledgements}

This work, supported by the European Communities under the contract of Association between EURATOM and CEA, was carried out within the framework of the European Fusion Development Agreement. The upgrade of the ECE radiometer in Tore Supra was partially funded by a "Région Provence-Côte d'Azur" grant under the PRIMAFLUTE (étude des Particules Rapides et Instabilités MAgnétohydrodynamiques par FLUctuations de Température Electronique) project.

\section{References}

1. J.L. Segui, et al, Rev. Sci. Instrum., 76, 123501 (2005)

2. D. Elbeze, et al., Proceedings of Electron Cyclotron Emission and Electron Cyclotron Resonance Heating (EC-15) workshop, p 220-225, Yosemite, USA (2009)

3. V.S. Udintsev et al., "Electron Temperature Fluctuation Studies in Different Confinement Regimes by Means of Correlation ECE on Tore Supra", In: ECA 29C, P-1.037, $32^{\text {nd }}$ EPS Conference on Plasma Phys., Tarragona, Spain (2005).

4. Z.O. Guimarães-Filho et al., Proceedings of $2^{\text {nd }}$ International Conference on Frontiers of Diagnostics, Frascati, Italy (2011)

5. A. Macor, et al., Phys. Rev. Lett. 102, 155005 (2009).

6. A. Merle, et al., submitted to Physics of Plasma (2012).

7. Z.O. Guimarães-Filho et al., submitted to Nuclear Fusion

8. Z.O. Guimarães-Filho et al., "Characterization of Energetic Particle Driven Modes by Spectral Analysis in Tore Supra and FTU tokamaks", AIP conference proceedings of the $5^{\text {th }}$ ITER International Summer School (2011). 